\title{
The geometry of genus-one helicoids
}

\author{
David Hoffman and Brian White*
}

\begin{abstract}
We prove: a properly embedded, genus-one, minimal surface that is asymptotic to a helicoid and that contains two straight lines must intersect that helicoid precisely in those two lines. In particular, the two lines divide the surface into two connected components that lie on either side of the helicoid. We prove an analogous result for periodic helicoid-like surfaces. We also give a simple condition guaranteeing that an immersed minimal surface with finite genus and bounded curvature is asymptotic to a helicoid at infinity.
\end{abstract}

Mathematics Subject Classification (2000). 53A10, 49Q05, 58E12.

Keywords. Complete embedded minimal surface, helicoid, variational methods.

\section{Introduction and statement of results}

In this paper we consider properly immersed minimal surfaces $\delta \subset \mathbb{R}^{3}$ that have one end asymptotic to the helicoid, and genus equal to one. We will call such a surface a nonperiodic genus-one helicoid. We are interested in embedded, nonperiodic genusone helicoids. Without loss of generality, we may assume that $\delta$ is asymptotic to a vertical helicoid whose axis is the $z$-axis, $Z$. If $\delta$ contains $Z$ and one horizontal line we will refer to $\delta$ as a symmetric, nonperiodic genus-one helicoid. Schwarz reflection ${ }^{1}$ about the lines on the surface provides the symmetries. Without loss of generality we may assume that this line is the $x$-axis, $X$, and that $\delta$ is asymptotic to the standard helicoid $H$, half of which is parametrized by

$$
(r, \theta) \rightarrow(r \cos \theta, r \sin \theta, \theta),
$$

$r \in[0, \infty), \theta \in \mathbb{R}$. (The other half is obtained by Schwartz reflection about $Z$.) Note that $X \cup Z \subset H$.

\footnotetext{
* The research of the first author was supported by the National Science Foundation, Division of Mathematical Sciences under grant DMS-0139410. The research of the second author was supported by the National Science Foundation, Division of Mathematical Sciences under grant DMS-0104049-NCX

${ }^{1} \mathrm{~A}$ minimal surface that contains a line in its interior is symmetric under reflection in that line; this symmetry is called Schwarz reflection and is a consequence of the Schwarz Reflection Principle in complex analysis. By the same token, a minimal surface that contains a straight line in its boundary can be continued analytically across that line by reflection. See [HK97] or [Oss86].
} 
Hoffman, Weber and Wolf [WHW06] proved the existence of a symmetric, embedded, nonperiodic genus-one helicoid. In [HW08] we gave a variational construction for such surfaces. The examples we constructed in that paper have the following property:

$8 \cap H=X \cup Z$, and

$8 \backslash H$ consists of two congruent simply connected components.

In Theorem 2.5 of Section 2.4, we prove that every embedded, symmetric, nonperiodic genus-one helicoid satisfies (2).

We also establish a parallel result for embedded periodic genus-one helicoids, by which we mean properly embedded minimal surfaces $S \subset \mathbb{R}^{3}$ that are invariant under a screw motion

$$
\sigma_{2 h}(r \cos \theta, r \sin \theta, z)=(r \cos (\theta+2 h), r \sin (\theta+2 h), z+2 h)
$$

for some $h>0$, and for which $S / \sigma_{2 h}$ has genus one and is asymptotic to $H / \sigma_{2 h}$ at infinity. Let $S^{*}=S \cap\{z:-h<z \leq h\}$, and note that $S^{*}$ is a fundamental domain for $S$. If $S$ contains $Z$ and if $S^{*}$ contains two horizontal lines, then we will refer to $S$ as a symmetric, periodic genus-one helicoid. Without loss of generality, we may assume that $X \subset S^{*}$, which implies that the other horizontal line in $S^{*}$ is $\sigma_{h}(X)$. For $h>\pi / 2$, such surfaces were proved to exist in [HKW99], [WHW06], and by variational means in [HW08]. Without loss of generality, we may assume that $\{z=h\} \cap S=\sigma_{h}(X)$, which implies that $S^{*}$ is bounded by two lines. This follows, for example, from Lemma 1(vii) of [WHW06] together with an application of the maximum principle. Define $X^{*}=X \cup \sigma_{h}(X)$. The construction in [HW08] produces periodic surfaces satisfying an analog of (2) above:

$$
\begin{aligned}
& S^{*} \cap H=X^{*} \cup Z^{*} \text {, and } \\
& S^{*} \backslash H \text { consists of two symmetric, simply connected components, }
\end{aligned}
$$

where $Z^{*}=\{(0,0, t):-h<t \leq h\}$. In this paper, we prove that every embedded, symmetric, periodic genus-one helicoid satisfies (4). This is Theorem 2.7.

We prove in Theorems 2.5 and 2.7 that embedded symmetric genus-one helicoids have simple intersections with all rotations of $H$ :

Let $\hat{H}$ be the result of rotating $H$ about the $z$-axis, $Z$ through an angle in $(0, \pi)$. If $\delta$ is nonperiodic, then $\delta \cap \hat{H}$ consists of $Z$ together with a smooth embedded closed curve that intersects $Z$ twice, once above and once below the $x y$-plane. If $S$ is periodic, $S^{*} \cap \hat{H}$ consists of $Z^{*}$ together with a smooth embedded closed curve that intersects $Z^{*}$ twice, once above and once below the $x y$-plane.

Section 2 concludes with a uniqueness result for half-helicoids, Theorem 2.11: Suppose $M$ is a connected minimal surface that lies in the closure of a component of $\mathbb{R}^{3} \backslash H$, with $\partial M$ lying in the closure of a component, $\Sigma$, of $H \backslash Z$. If $M$ is bounded 
or if $M$ is asymptotic to $\Sigma$, then $M \subset \Sigma$. The proof of this result uses the fact that $\mathbb{R}^{3} \backslash Z$ is foliated by half-helicoids. Our approach is close to that taken by Hardt and Rosenberg in [HR90].

As mentioned above, [WHW06] and [HW08] proved existence of $\sigma_{2} h$-invariant, symmetric genus-one helicoids for every $h>\pi / 2$. In Theorem 3.4 of Section 3.3, we prove that the condition $h>\pi / 2$ is necessary: for $h \leq \pi / 2$, there are no embedded, symmetric, periodic genus- $g$ helicoids (with $g \geq 1$ ) invariant under the screw motion $\sigma_{2} h$. To our knowledge, this was first observed by Bill Meeks for $h<\pi / 2$. Our result requires only the presence of two horizontal lines in $S / \sigma_{2 h}$ (no assumption that $S$ contains the axis $Z$ ). This proof uses Proposition 5.1, which gives estimates of the radial decay of the vertical distance between the end of a symmetric, periodic genus-one helicoid and the end of a helicoid. We use the same estimates to prove (Theorem 5.4) that the $\sigma_{2 h}$-invariant, helicoid-like surfaces constructed in [HW08] are asymptotic to helicoids and thus are in fact periodic genus-one helicoids.

In Section 4, we investigate the geometry of properly immersed minimal surfaces with finite genus and one end. With a few additional assumptions, we prove that such a surface is asymptotic to a helicoid:

Let $\delta \subset \mathbb{R}^{3}$ be a properly immersed minimal surface with finite genus, one end and bounded Gauss curvature. Suppose that $\$$ contains $X \cup Z$, and that one level set $\left\{x_{3}=c\right\} \cap 8$ has precisely one divergent component and a finite number of singular points. Then 8 is conformally a compact Riemann surface punctured in one point corresponding to the end, and that end is asymptotic to a helicoid.

This is Theorem 4.1. This result gives another proof that the genus-one surfaces constructed in [HW08] are asymptotic to the helicoid. The method of proof here is a slight generalization of the method used in that paper. (See Theorem 6.1 in [HW08].)

\section{Structural properties of symmetric genus-one helicoids}

An embedded, nonperiodic genus-one helicoid $\delta \subset \mathbb{R}^{3}$ is a properly embedded minimal surface in $\mathbb{R}^{3}$ that is asymptotic to the helicoid at infinity. Without loss of generality, we will assume that $\delta$ is asymptotic to the helicoid $H$ defined in the first paragraph of the Introduction. The surface $H$ is a right-handed helicoid that contains $Z$ and $X$. We say that $\delta$ is symmetric if it contains $Z$ and $X$. Similarly, an embedded, periodic genus-one helicoid is a properly embedded minimal surface $S \subset \mathbb{R}^{3}$ invariant under a screw motion (3), such that $S / \sigma_{2 h}$ has genus one and two helicoidal ends. We say that $S$ is symmetric if $Z \subset S$, and the fundamental domain $S^{*}=S \cap\{-h<z \leq h\}$ contains $X^{*}=X \cup \sigma_{h}(X)$.

In this section we will prove that embedded, symmetric genus-one helicoids are cut by $H$ precisely along $X \cup Z$ into two congruent simply connected domains. 
We also prove the analogous result for periodic genus-one helicoids. The technique involves the study of minimal surfaces with boundary lying in a half-helicoid.

2.1. Removal of the axes results in two congruent, simply connected domains. We begin by showing that removal of $X \cup Z$ from a properly embedded, nonperiodic, symmetric genus-one helicoid produces two congruent, simply connected domains. Similarly, removal of $X^{*} \cup Z^{*}$ from a fundamental domain of a properly embedded, periodic, symmetric genus-one helicoid also produces two congruent simply connected domains.

Lemma 2.2. Suppose 8 is a properly embedded, nonperiodic symmetric genus-one helicoid. Then $\delta \backslash Z$ and $\delta \backslash X$ are annuli, and $\delta \backslash(X \cup Z)$ is a pair of congruent, simply connected domains.

Lemma 2.3. Suppose $S$ is a properly embedded, periodic, symmetric genus-one helicoid invariant under $\sigma_{2 h}$. Let $S^{*}=\{-h<z \leq h\}$ be a fundamental domain of $S$, and $Z^{*}=Z \cap\{-h<z \leq h\}$. Then $S^{*} \backslash Z^{*}$ and $S^{*} \backslash X^{*}$ are annuli, and $S^{*} \backslash\left(Z^{*} \cup X^{*}\right)$ is a pair of congruent, simply connected domains.

Proof of Lemma 2.2. The surface $\&$ is topologically a once-punctured torus. (In fact by Theorem $4.1, \delta$ is conformally a once-punctured torus, but we will not use that here.) Thus the one-point compactification $T=\delta \cup\{\infty\}$ is a torus and $Z \cup\{\infty\}$ is a simple closed curve in $T$. Removing a simple closed curve from a torus either separates it into a disk and a once-punctured torus, or else results in a single annulus.

The rotation $\rho_{Z}$ is an isometry of $\delta$ that leaves $Z$ invariant. Therefore, $Z$ cannot divide $T$ into a disk and punctured torus, because these pieces would have to be homeomorphic (by the involution $\rho_{Z}$ ). Thus the result is a single annulus $A$.

For similar reasons, $X$, viewed as a curve in $T$, is a simple closed curve. The same argument shows that removal of $X$ from $T$ produces an annulus.

Note that $Z$ and $X$ cross at the origin and at the point at infinity. These two points are represented as points on the boundary of the annulus $A=T \backslash Z$. The positive ray of $X$ is a simple curve in $A$ going from one boundary point to another. If it went from one boundary component of $A$ to the same boundary component, it would divide $A$ into two components, one a disk, the other an annulus. But if there were two components, they would be homeomorphic (by $\rho_{X}$ ). Thus, the positive ray of $X$ goes from one boundary component of $A$ to the other, and removing it results in a disk. Now removing the negative ray of $X$ divides that disk into two disks. The two disks are congruent since they are related by the isometry $\rho_{X}$

Proof of Lemma 2.3. Note that the one-point compactification of $S \cap\{-h<z<h\}$ is a torus $T$. The proof of Lemma 2.3 is exactly the same as the proof of Lemma 2.2, except that one replaces $\delta$ and $Z$ by $S \cap\{-h<z<h\}$ and $Z \cap\{-h<z<h\}$. 
2.4. The decomposition theorem for nonperiodic symmetric genus-one helicoids. The helicoid $H$ divides $\mathbb{R}^{3}$ into two simply connected regions. Let $H^{+}$be the region that contains $Y^{+}$, the positive ray of the $y$-axis, and let $H^{-}$be the other region. The axis $Z$ is contained in the helicoid $H$, and $H \backslash Z$ consists of two simply connected components, each of which is we will refer to as a half helicoid. We will denote by $\Sigma_{0}$ the half-helicoid that contains the $X^{+}$, the positive $x$-axis.

More generally, let us extend the definition of a half-helicoid to include any surface obtained by rotating one of the components of $H \backslash Z$ through some angle about $Z$. Thus the half-helicoids form a foliation of $\mathbb{R}^{3} \backslash Z$. In particular, rotating $\Sigma_{0}$ through angles in $(0, \pi)$ produces a foliation of $H^{+}$.

Theorem 2.5. Let 8 be an embedded, nonperiodic, symmetric genus-one helicoid. Then

1. $\delta \backslash(X \cup Z)$ consists of two simply connected, congruent components $D$ and $D^{\prime}$.

2. $D$ and $D^{\prime}$ lie in $\mathbb{R}^{3} \backslash H$, one in $H^{+}$, the other in $H^{-}$.

3. Let $\hat{H}$ be a helicoid obtained by rotating $H$ about $Z$ through an angle in $(0, \pi)$. Then $D \cap \hat{H}$ (resp. $D^{\prime} \cap \hat{H}$ ) is a smooth embedded curve with one endpoint in $Z^{+}$and the other endpoint in $Z^{-}$.

Proof. Statement 1 is Lemma 2.2. It remains to prove statements 2 and 3. We begin by observing that by assumption $\delta$ contains the axes $X$ and $Z$ and $\partial D=\partial D^{\prime}=X \cup Z$. Let $\rho_{X}$ and $\rho_{Z}$ denote rotations by $\pi$ about $X$ and $Z$, respectively. These symmetries are orientation-reversing on $\delta$, and it is easy to see that they interchange $D$ and $D^{\prime}$. It follows that their composition, $\rho_{Y}=\rho_{X} \circ \rho_{Z}$, rotation by $\pi$ about the axis $Y$, leaves $D$ and $D^{\prime}$ invariant and preserves orientation on $\&$.

If $p \in \mathbb{R}^{3} \backslash Z$, let $\Sigma(p)$ be the half-helicoid that contains $p$. If $p \in Z \backslash\{0\}$, let $\Sigma(p)$ be the half-helicoid with the property that $\Sigma(p)$ and $D$ have the same tangent half-plane at $p$. Note that

If $p \in Z \backslash\{0\}$, then $D \cap \Sigma(p)$ contains a smooth curve, one of whose endpoints is $p$.

Since $\bar{D} \backslash Z$ is simply connected, there is a smooth function

$$
\theta: \bar{D} \backslash Z \rightarrow \mathbb{R}
$$

such that

$$
q=(x, y, z)=(r \cos \theta, r \sin \theta, z)
$$

for $q \in \bar{D} \backslash Z$, where $r=r(x, y, z)=\sqrt{x^{2}+y^{2}}$. We may normalize $\theta$ so that

$$
\theta=0 \text { on } X^{+} \text {, }
$$


where $X^{+}$is the positive $x$-axis. It follows that for some integer $k$,

$$
\theta=(2 k+1) \pi \quad \text { on } X^{-},
$$

where $X^{-}=\rho_{Y}\left(X^{+}\right)$, the negative $x$-axis. Since $\bar{D} \backslash\{0\}$ is a smooth manifold with boundary $X \cup Z \backslash\{0\}$, the function $\theta$ extends smoothly to $Z \backslash\{0\}$. Thus $\theta$ is a smooth function on $\bar{D} \backslash\{0\}$.

Since $\theta=0$ on $X^{+}$and $\theta=(2 k+1) \pi$ on $X^{-}$, extending $\theta$ to 0 is somewhat problematic. We get around that by using the geodesic completion $D^{*}$ of $\bar{D} \backslash\{0\}$. Note that $D^{*}$ is $\bar{D} \backslash\{0\}$ together with two points, $0^{+}$and $0^{-}$, which are the limits of $(x, 0,0)$ as $x \downarrow 0$ and $x \uparrow 0$, respectively. We let

$$
\theta\left(0^{+}\right)=0 \text { and } \theta\left(0^{-}\right)=(2 k+1) \pi,
$$

which makes $\theta$ continuous on all of $D^{*}$.

We now use the $\rho_{Y}$ symmetry of $D$. Since $\rho_{Y}(x, y, z)=(-x, y,-z)$,

$$
\cos \left(\theta \circ \rho_{Y}\right)=-\cos \theta \quad \text { and } \quad \sin \left(\theta \circ \rho_{Y}\right)=\sin \theta,
$$

from which it follows that $\theta \circ \rho_{Y}$ and $-\theta$ differ by an odd multiple of $\pi$. In fact, since $\rho_{Y}\left(X^{+}\right)=X^{-}$, it follows from (7) and (8) that

$$
\theta \circ \rho_{Y}=-\theta+(2 k+1) \pi .
$$

Since $D$ is a disk and $\rho_{Y}$ is an orientation-preserving isometric involution of $\mathrm{D}, \rho_{Y}$ has a unique fixed point. That is, $D \cap Y$ is a single point. Without loss of generality, we can assume that this fixed point is in $H^{+}$. (Since $D^{\prime}=\rho_{Z}(D)$, we can simply relabel the disks.) In particular,

$$
D \cap Y^{-}=\emptyset,
$$

where $Y^{-}$is the negative $y$-axis. Let

$$
F: D \rightarrow \mathbb{R}, \quad(x, y, z) \mapsto \theta(x, y, z)-z .
$$

The function $F$ is connected to the geometry of our situation by the following elementary observation:

$$
F \text { is constant on every half-helicoid. }
$$

Claim 1. F has no local maxima or local minima on $D$.

Proof of Claim 1. Suppose $F$ has a local maximum or minimum at $p \in D$. Let $\mathbb{B}$ be a ball centered at $p$, small enough so that $\mathbb{B}$ is disjoint from $Z$ and that $D \cap \mathbb{B}$ is connected. Then there is a unique continuous extension of $\theta$ to $D \cup \mathbb{B}$ so that (6) still 
holds. Note that if we use this extended $\theta$ together with (12) to define $F$ on $\mathbb{B}$, then it follows from (13) that

$$
\{q \in \mathbb{B}: F(q)=F(p)\}=\Sigma(p) \cap \mathbb{B} .
$$

Thus $D \cap \mathbb{B}$ lies in the closure of one of the connected components of $\mathbb{B} \backslash \Sigma(p)$, and $D \cap \mathbb{B}$ and $\Sigma(p) \cap \mathbb{B}$ are tangent at $p$. By the maximum principle, $D \cap \mathbb{B}$ and $\Sigma(p) \cap \mathbb{B}$ coincide. By analyticity, all of $D$ is contained in a helicoid, a contradiction.

Claim 2. $F$ has no local maxima or local minima on $Z \backslash\{0\}$.

Proof of Claim 2. Suppose $p \in Z \backslash\{0\}$. Since $\Sigma(p)$ and $\bar{D} \backslash\{0\}$ are minimal surfaces with boundary and since they are tangent at $p, D \cap \Sigma(p)$ contains a smooth curve $C$ with $p$ as one of its endpoints. Note that $F$ is constant along $C$ by (13). By Claim 1, none of the points of $C$ is a local maximum or local minimum of $F$. Thus $p$ is neither a local maximum nor a local minimum of $F$.

Claim 3. Suppose $\alpha$ is not an integral multiple of $\pi$. Then either $F^{-1}(\alpha)$ is empty, or it is a single smooth curve with one endpoint on $Z^{+}$and the other endpoint on $Z^{-}$.

Proof of Claim 3. Suppose $p \in C=F^{-1}(\alpha)$. Then $C \subset \Sigma(p)$. Note that $D$ is asymptotic to $H$ at infinity and $\Sigma(p)$ is not (because $\alpha$ is not an integral multiple of $\pi$ ), so $C$ lies in a bounded region of $\mathbb{R}^{3}$. Now $C$ cannot contain a closed curve, because then that curve would bound a region in $D$, and $F$ would have an interior maximum or minimum in that region, violating Claim 1. Thus each connected component $T$ of $C$ has the structure of a tree whose endpoints are on $X \cup Z$. Since $F=0$ on $X^{+}$ and $F=(2 k+1) \pi$ on $X^{-}$, the endpoints must be on $Z$. Since 0 and $(2 k+1) \pi$ are the only subsequential limits of $F(p)$ as $p \rightarrow 0$, in fact the endpoints of $T$ must be on $Z \backslash\{0\}$.

Now $T$ cannot have two endpoints on $Z^{+}$. For if it did, $T$ would contain a curve $\Gamma$ joining those endpoints, and that curve together with the interval $I \subset Z$ joining the endpoints would bound a region $U$ in $D$. Since $F$ is constant on $\Gamma, F$ would have a local maximum or a local minimum at some point $p \in U \cup I$. But that is impossible by Claims 1 and 2 .

Thus $T$ has at most one endpoint on $Z^{+}$and (by the same reasoning) at most one endpoint on $Z^{-}$. It follows that $T$ is a smooth curve joining a point on $Z^{+}$to a point on $Z^{-}$.

We have shown: $C=F^{-1}(\alpha)$ is a union of disjoint curves, each of which joins a point in $Z^{+}$to a point in $Z^{-}$. Furthermore, there cannot be more than one such curve. For if there were two such curves $T_{1}$ and $T_{2}$, then those curves together with a pair of intervals $I^{+}$and $I^{-}$(in $Z^{+}$and $Z^{-}$, respectively) would bound a region $U$. Since $F$ is not constant on $\bar{U}$, its maximum on $\bar{U}$ is greater than $\alpha$ or its minimum on 
$\bar{U}$ is less than $\alpha$. Thus $F$ has a local maximum or a local minimum on $U \cup I^{+} \cup I^{-}$. But that is impossible by Claims 1 and 2 .

Claim 4. If $p \in Y^{-}$, then $D \cap \Sigma(p)=\emptyset$.

Proof of Claim 4. Note that $D \cap \Sigma(p)$ is the union of $F^{-1}(\alpha)$ over all $\alpha$ that are congruent to $3 \pi / 2 \bmod 2 \pi$. Thus if $D \cap \Sigma(p)$ were nonempty, by Claim 3 it would contains a curve joining $Z^{+}$to $Z^{-}$. But any such curve in $\Sigma(p)$ must cross $Y^{-}$, and $D$ does not contain any points in $Y^{-}$. Thus $D \cap \Sigma(p)=\emptyset$.

By Claim 4 , the set $F(D)$ does not contain any values equal to $3 \pi / 2 \bmod 2 \pi$. In particular, it contains neither $-\pi / 2$ nor $3 \pi / 2$. Since $D$ is connected, $F(D)$ is an interval. Since $F \mid D$ has no local maxima or local minima, $F(D)$ is an open interval $(a, b)$. From (9), $[a, b]$ contains 0 and $(2 k+1) \pi$. Thus

$$
F(D)=(a, b), \quad \text { where }-\pi / 2 \leq a \leq 0 \text { and } \pi \leq b \leq 3 \pi / 2,
$$

and $k=0$. In particular, from (10) we have

$$
\theta \circ \rho_{Y}=-\theta+\pi .
$$

Claim 5. $a=0$ and $b=\pi$.

Proof of Claim 5. If $b \neq \pi$, then by (14), $\pi<b \leq 3 \pi / 2$. Let $b_{i} \in(\pi / 2, b)$, $b_{i} \neq \pi$, with $b_{i} \rightarrow b$. Then $F^{-1}\left(b_{i}\right)$ is nonempty, and by Claim 3, it must contain a point $p_{i}$ in the $x y$-plane. Note that $p_{i}$ has the form

$$
p_{i}=\left(r_{i} \cos b_{i}, r_{i} \sin b_{i}, 0\right),
$$

where $r_{i}>0$. By passing to a subsequence, we may assume that the $r_{i}$ converge to a limit $r \in[0, \infty]$. Now $r$ cannot be 0 since $\theta\left(0^{+}\right)=0$ and $\theta\left(0^{-}\right)=\pi$. Also, $r$ cannot be a finite nonzero number since otherwise $F$ would attain its maximum, contradicting Claim 1.

Finally, if $r_{i} \rightarrow \infty$, then $\operatorname{dist}\left(p_{i}, H\right) \rightarrow b-\pi>0$, which is impossible since $D$ is asymptotic to $H$ at infinity. The contradiction proves that $b=\pi$. The proof that $a=0$ is essentially the same. (It also follows from the $\rho_{Y}$ symmetry of $D$.)

Since $F(D)=(0, \pi)$, it follows that $D$ intersects only those half-helicoids produced by rotating $\Sigma_{0}$ through an angle in $(0, \pi)$. As observed in the paragraph just before the statement of the theorem, those half-helicoids foliate $\mathrm{H}^{+}$, so $D$ lies in $H^{+}$. It follows that $D^{\prime}=\rho_{Y} D$ lies in $H^{-}=\rho_{Y} H^{+}$. This completes the proof of statement (2).

Statement (3) of the theorem follows from Claim 3, together with the fact that $F(D)=(0, \pi)$. 
2.6. The decomposition theorem for periodic symmetric genus-one helicoids. There is an analogous result to Theorem 2.5 for periodic, symmetric genus-one helicoids.

Theorem 2.7. Let $S$ be a periodic, embedded, symmetric genus-one helicoid. Then

1. $S^{*} \backslash\left(X^{*} \cup Z^{*}\right)$ consists of two simply connected components $D$ and $D^{\prime}$.

2. $D$ and $D^{\prime}$ lie in $\mathbb{R}^{3} \backslash H$, one in $H^{+}$the other in $H^{-}$.

3. Let $\hat{H}$ be a helicoid obtained by rotating $H$ about $Z$ through an angle in $(0, \pi)$. Then $D \cap H^{\prime}$ (resp. $\left.D^{\prime} \cap \hat{H}\right)$ is a smooth embedded curve with one endpoint in $Z^{+}$and the other endpoint in $Z^{-}$.

The sets $S^{*}$ and $X^{*}$ are defined in the Introduction, just before equation (4). The proof of Theorem 2.6 is a straightforward adaptation of the proof of Theorem 2.5. (Statement 1 was already proved in Lemma 2.2.)

2.8. A half-helicoid uniqueness theorem. We end this section with a uniqueness theorem for minimal surfaces that lie in the closure of a component of $\mathbb{R}^{3} \backslash H$, and that are either compact or asymptotic to $H$ at infinity.

Definition 2.9. Let $S$ be an unbounded, oriented, embedded surface in $\mathbb{R}^{3}$. We say that another surface $M$ is asymptotic to $S$ at infinity provided there is a domain $\Omega \subset S$ and a function $u: \Omega \rightarrow \mathbb{R}$ such that

$$
\lim _{|p| \rightarrow \infty}(|u(p)|+|D u(p)|)=0
$$

and such that outside of a compact subset of $\mathbb{R}^{3}$, the surface $M$ coincides with the graph

$$
\{p+u(p) v(p): p \in \Omega\},
$$

where $v(p)$ is the unit normal to $S$ at $p$.

Remark 2.10. If $M$ and $S$ have compact boundaries and bounded principal curvatures, then the $C^{1}$ condition (16) follows (by an Arzela-Ascoli type argument) from the analogous $C^{0}$ condition $\lim _{|p| \rightarrow \infty}|u(p)|=0$.

Theorem 2.11. Let $\Sigma$ be one of the components of $H \backslash Z$. Suppose $M$ is a connected minimal surface in $\overline{H^{+}} \backslash Z$ such that $\partial M \subset \bar{\Sigma}$ and such that $M$ is either bounded or asymptotic to $\Sigma$ at infinity.

Then $M$ is a subset of $\Sigma$. 
Proof. We may assume without loss of generality that $\Sigma$ is the component of $H \backslash Z$ containing the positive $x$-axis. We may also assume that $M$ is bounded: If $M$ is not bounded then by hypothesis $M$ is asymptotic to $\Sigma$ at infinity. A sufficiently small rotation of $M$ about $Z$, say through an angle $-\epsilon$, will produce a surface with nonempty intersection with $H^{+}$. Since $M$ is asymptotic to $\Sigma \subset H$ at infinity, the intersection of the rotated $M$ with $H^{+}$is bounded. Denote this intersection by $M(\epsilon)$. If $M$ were a counterexample to the theorem, then the surface $M(\epsilon)$ would also be a counterexample, a bounded one.

Thus from now on we assume that $M$ is bounded.

Let $C$ be a closed, solid circular cylinder of finite height that contains $M$ and whose axis of symmetry is $Z$. Let $\Gamma$ be the boundary of $C \cap \Sigma$. Minimize area among disks in $\overline{H^{+}}$with boundary $\Gamma$ and with $M$ as an obstacle (i.e., among disks $\Delta$ such that $M$ is contained in the closed region bounded by $\Delta \cup(C \cap \Sigma)$.) Call the resulting disk $D$.

Let $\Sigma^{\prime}$ be the half-helicoid $H \backslash(Z \cup \Sigma)$. Rotate $\Sigma^{\prime}$ in $H^{+}$until it touches $D$ at an interior point or until it becomes tangent to $D$ at some point of the interval $I:=\Gamma \cap Z$. Call the resulting half-helicoid $\Sigma^{*}$.

Note that one of the following must occur:

1. $\Sigma^{*}$ touches $D$ at an interior point of $D$.

2. $\Sigma^{*}$ is tangent to $D$ at an interior point of $I$.

3. $\Sigma^{*}$ is tangent to $D$ at an endpoint of $I$.

In case $1, D$ is contained in $\Sigma^{*}$ by the maximum principle. In case $2, D$ is contained in $\Sigma^{*}$ by the boundary maximum principle. In case $3, \Sigma^{*}=\Sigma$ (since $D$ and $\Sigma$ are tangent at the endpoints of $I$ ) and thus $D$ is contained in $\Sigma^{*}$.

In all three cases, we have shown that $D$ is contained in $\Sigma^{*}$. Since $\partial D \subset \Sigma$, this implies that $\Sigma^{*}=\Sigma$. Since $M$ lies between $\Sigma$ and $\Sigma^{*}$, in fact $M$ is contained in $\Sigma$.

\section{Nonexistence of embedded, periodic, higher genus helicoids with small twist angles}

In this section we study properly embedded, periodic minimal surfaces invariant under a screw motion $\sigma_{2 h}$ and asymptotic to the helicoid. We will show that if $h \leq \pi / 2$ and if the intersection of the surface with some horizontal plane is a line, then the surface must be the helicoid.

3.1. The total curvature of almost-helicoidal curves. The curvature of a space curve $\theta \mapsto c(\theta)$ is given by $\frac{\left|c^{\prime}(\theta) \times c^{\prime \prime}(\theta)\right|}{\left|c^{\prime}(\theta)\right|^{3}}$, and therefore the total curvature from 
$\theta=0$ to $\theta=A$ is

$$
\int_{c} k d s=\int_{0}^{A} \frac{\left|c^{\prime}(\theta) \times c^{\prime \prime}(\theta)\right|}{\left|c^{\prime}(\theta)\right|^{2}} d \theta
$$

Now suppose that

$$
c(\theta)=(r \cos \theta, r \sin \theta, \theta+f(r, \theta))
$$

for some function $f(r, \theta)$. In the special case that $f$ is constant, the curve $c$ is a standard helix. More generally, suppose that $\frac{\partial f}{\partial \theta}$ and $\frac{\partial^{2} f}{\partial \theta^{2}}$ tend to zero uniformly as $r \rightarrow \infty$. Since $\left|c^{\prime}(\theta)\right|^{2}=r^{2}+\left(1+\frac{\partial f}{\partial \theta}\right)^{2}$ and $\left|c^{\prime \prime}(\theta)\right|^{2}=r^{2}+\left(\frac{\partial^{2} f}{\partial \theta^{2}}\right)^{2}$, it follows that

$$
\left|c^{\prime \prime}(\theta)\right|<\left|c^{\prime}(\theta)\right|
$$

for $r$ sufficiently large. Therefore

$$
\frac{\left|c^{\prime}(\theta) \times c^{\prime \prime}(\theta)\right|}{\left|c^{\prime}(\theta)\right|^{2}} \leq \frac{\left|c^{\prime}(\theta)\right|\left|c^{\prime \prime}(\theta)\right|}{\left|c^{\prime}(\theta)\right|^{2}}<\frac{\left|c^{\prime}(\theta)\right|^{2}}{\left|c^{\prime}(\theta)\right|^{2}}=1
$$

for $r$ sufficiently large. It follows that the total curvature (17) is strictly less than $A$. We state this observation as a lemma.

Lemma 3.2. Let $f(r, \theta)$ be a function defined for $r \geq R_{0}$ and $\theta \in[0, A]$. Suppose that $\frac{\partial f}{\partial \theta}$ and $\frac{\partial^{2} f}{\partial \theta^{2}}$ tend to zero uniformly as $r \rightarrow \infty$. Then for every sufficiently large $r$, the curve

$$
\theta \in[0, A] \mapsto(r \cos \theta, r \sin \theta, \theta+f(r, \theta))
$$

has total curvature strictly less than A.

3.3. Nonexistence of examples with $\boldsymbol{h} \leq \boldsymbol{\pi} / \mathbf{2}$. We will use Lemma 3.2 to prove

Theorem 3.4. Let $S$ be a properly immersed minimal surface that lies in the slab $\{-h \leq z \leq h\}$ and that is bounded by the two lines $H \cap\{z=h\}$ and $H \cap\{z=-h\}$, where $H$ is the standard helicoid (1). Suppose that $S$ is asymptotic ${ }^{2}$ to

$$
H \cap\{-h \leq z \leq h\} .
$$

If $h \leq \pi / 2$, then $S=H \cap\{-h \leq z \leq h\}$.

As a corollary we have

Theorem 3.5. Suppose that $S$ is a properly immersed minimal surface invariant under a screw motion $\sigma_{2 h}$ with $h \leq \pi / 2$, and that $S$ is asymptotic to $H$ as $r=\sqrt{x^{2}+y^{2}}$ tends to infinity. If the intersection of $S$ with some horizontal plane is a line, then $S=H$.

\footnotetext{
${ }^{2}$ See Definition 2.9 .
} 
Proof. Without loss of generality we may assume that $S \cap\{z=-h\}$ is a line. By the $\sigma_{2 h}$ invariance, $S \cap\{z=h\}$ must also be a line. Since $S$ is asymptotic to $H$ away from $Z$, these lines must be the lines $H \cap\{z=-h\}$ and $H \cap\{z=h\}$. Thus $S \cap\{-h \leq z \leq h\}$ satisfies the hypotheses of Theorem 3.4, from which we conclude that $S=H$.

Proof of Theorem 3.4. By assumption, outside of any sufficiently large cylinder $C_{R}=$ $\left\{x^{2}+y^{2} \leq R^{2},|z| \leq h\right\}$, one end of $S$ is a graph of the form

$$
\{(r \cos \theta, r \sin \theta, \theta+f(r, \theta)):-h \leq \theta \leq h, r>R\},
$$

with $f(-h, r)=f(h, r)=0$. (A similar discussion applies to the other end. Indeed, after rotation by $\rho_{Z}$, the other end has the same form.) By Proposition 5.1 (20),

$$
\left|\frac{\partial w}{\partial \theta}\right|=o\left(r^{-\beta}\right), \quad \text { and } \quad\left|\frac{\partial^{2} w}{\partial \theta^{2}}\right|=o\left(r^{-\beta}\right)
$$

for every $\beta<\pi / 2 h$. Since $h \leq \pi / 2$, these estimates hold for every $\beta<1$.

Let $S_{R}=S \cap C_{R}$ be the portion of $S$ inside the cylinder $C_{R}$. Note that $\partial S_{R}$ is an extremal curve: it lies on the boundary of the convex set $C_{R} \cap\{|z| \leq h\}$.

Claim. For $R$ sufficiently large, the total curvature of $\partial S_{R}$ is strictly less than $4 \pi$.

Proof of Claim. The curve $\partial S_{R}$ consists of two line segments-one on the top and one on the bottom disk of $\partial C_{R}$-and two nearly helical curves on $\partial C_{R}$. There are four corners where the curves and the line segments meet orthogonally.

By Lemma 3.2, each of the two nearly helical arcs has total curvature strictly less than $2 h$, provided $R$ is sufficiently large. Thus $\partial S_{R}$ has total curvature strictly less than

$$
2(2 h)+4(\pi / 2)=4 h+2 \pi \leq 4 \pi
$$

since $h \leq \pi / 2$ and since each corner contributes $\pi / 2$ to the total curvature.

We now apply the following uniqueness result :

Theorem 3.6. A smooth, extremal Jordan curve with total curvature at most $4 \pi$ bounds precisely one minimal surface and that minimal surface is an embedded disk.

Meeks and Yau [MY82] prove that a smooth, extremal Jordan curve either bounds two distinct embedded minimal disks or it bounds a unique minimal disk and no other minimal surface of any genus. Together with the result of Nitsche [Nit73] that a smooth Jordan curve with total curvature not greater than $4 \pi$ bounds a unique minimal disk, they arrive at Theorem 3.6.

The curve $\partial S_{R}$ we are dealing with has four corners that can be smoothed in the surface with an arbitrarily small increase in total curvature, so that the smoothed 
curve will also have total curvature strictly less than $4 \pi$. Hence by Theorem 3.6 (applied to the smoothed curve), $S_{R}$ is simply connected for all sufficiently large $R$, and therefore $S$ is simply connected.

Thus the surface obtained from $S$ by repeated Schwarz reflection about the boundary lines is a nonplanar, singly periodic, embedded and simply connected minimal surface. By a theorem of Meeks and Rosenberg [MR93], the only such surface is the helicoid.

Remark 3.7. For $h<\pi / 2$, it is also possible to prove Theorem 3.4, without recourse to Theorem 3.6, as follows. The decay estimates of Proposition 5.1 can be used to show that (for $R$ sufficiently large) the curve $\partial S_{R}$ projects monotonically to the boundary of a convex region $\Omega$ in the plane $\{x=0\}$. (One shows that the curvature at each point of the projections of the perturbed helical arcs is strictly positive.) By a theorem of Rado (see for example Sections 398 and 400 in Nitsche [Nit89]), $S_{R}$ must be a graph over $\Omega$. In particular, $S_{R}$ must be simply connected.

If $h=\pi / 2$, the curve $\partial S_{R}$ still projects monotonically to the boundary of a region $\Omega$ in the plane $\{x=0\}$. However, convexity of $\Omega$ at the projections of the corner points of $\partial S_{R}$ seems to be delicate. In particular, the convexity does not seem to follow from the decay estimates in Proposition 5.1.

\section{Asymptotic behavior of symmetric, properly immersed minimal surfaces with one end and finite topology}

The goal of this section is to prove the following theorem:

Theorem 4.1. Let $\delta \subset \mathbb{R}^{3}$ be a properly immersed, nonplanar minimal surface with finite genus, one end, and bounded curvature. Suppose

1. 8 contains $X \cup Z$, and

2. for some value of $c,\left\{x_{3}=c\right\} \cap \&$ has precisely one divergent component.

Then 8 is conformally a once-punctured Riemann surface, and 8 is asymptotic to a helicoid.

Note that any level set $M \cap\{z=c\}$ of a properly embedded minimal surface $M \subset \mathbb{R}^{3}$ can be decomposed uniquely as a union of connected $C^{1}$, properly immersed curves, all intersections and self-intersections of which are transverse. The intersection points are precisely the points of tangency of $M$ and the plane $\{z=c\}$. Thus hypothesis 2 of Theorem 4.1 is equivalent to: there are only finitely many points of tangency of $\{z=c\}$ and $M$, and $M \cap\{z=c\}$ can be written as the union of finitely many connected $C^{1}$ immersed curves, exactly one of which is not closed. 
Remark 4.2. Our proof of Theorem 4.1 is similar to the proof in [HW, §6.1] that the surfaces constructed in that paper are conformally punctured tori and are asymptotic to a helicoid at infinity.

Proof of Theorem 4.1. The surface $\delta$ satisfies the hypotheses of the following theorem of Rodriguez and Rosenberg [RR98]:

Suppose $\delta \subset \mathbb{R}^{3}$ is a properly immersed minimal surface with one end and with bounded curvature. Suppose also that at some level $x_{3}=c$, the intersection $\left\{x_{3}=c\right\} \cap 8$ consists of finitely many curves with finitely many intersections. Then 8 is of finite type, i.e., 8 is conformally a once-punctured Riemann surface, the puncture corresponding to the end, and the one-forms $d g / g$ and $d h$ are meromorphic on the compact surface.

Here, $g$ is the stereographic projection of the Gauss map from the north pole, and $d h=d x_{3}+i d x_{3}^{*}$ is a holomorphic one form on $M$. (The function $x_{3}^{*}$ is a harmonic conjugate of $x_{3}$; it is locally well-defined up to an additive constant.) Note that $d h$ is closed but is not, in general, exact.

Claim. The one form $d h$ has a double pole at the puncture and no residue. The one form $d g / g$ also has a double pole at the puncture.

Assuming the claim, we can complete the proof of the theorem by using the following result of Hoffman and McCuan [HM03]: Let $E \subset \mathbb{R}^{3}$ be a properly immersed, minimal annular end that is conformally a punctured disk. Suppose that $d g / g$ and $d h$ both have double poles at the puncture and that $d h$ has no residue at the puncture. If $E$ contains a vertical ray and a horizontal ray, then $E$ is asymptotic to a helicoid at infinity.

We apply this theorem to an end $E$ of 8 corresponding to a neighborhood of the puncture. That $E$ contains the requisite rays follows from assumption 1 of the theorem.

Proof of Claim. By assumption 2, in a neighborhood of the puncture, the level curve $\left\{x_{3}=c\right\} \cap 8$ consists of two smooth curves emanating from the puncture. Hence $d h$ has a pole of order two at that point. (That $d h$ must have a pole at the puncture follows from the maximum principle and the fact that $\delta$ has one end.) Since $d h$ is holomorphic on $S$, it follows from Stokes' Theorem that $d h$ has no residue at the puncture. In a (possibly smaller) neighborhood of the puncture, $d h$ can be assumed to have no zeros. Since $d h$ has a double pole at the puncture, the level curves $\left\{x_{3}=a\right\} \cap \delta$, for any value of $a$ are embedded in this neighborhood. In particular, $\delta$ has an embedded end.

We claim that $d g / g$ must have a pole at the puncture. For suppose it does not. Then $g$ has a well-defined value at the puncture, and $g$ is meromorphic on the onepoint compactification of $\delta$. Thus $\delta$ has finite total curvature ([Oss63], [Oss86], Chapter 9, [HK97], Section 2.3) and, as observed above, an embedded end. Such 
ends are asymptotic to a plane or to an end of the catenoid ([Sch83] , [LM83], [HK97], Section 2.3). On a catenoid $C, d h$ has a simple pole at an end (observe that the level curves $\left\{x_{3}=a\right\} \cap C$ are circles that do not pass through the point corresponding to the end.) Therefore, $\delta$ is asymptotic to a plane, and since it has one end, the maximum principle implies that it is equal to a plane. Since we are assuming that $\delta$ is nonplanar, the contradiction shows that $d g / g$ has a pole at the puncture.

We now determine the order of the pole of $d g / g$ at the puncture. First of all we will show that the order of the pole is even. Note that on the compact Riemann surface $\delta \cup\{\infty\}$, the number of zeros minus the number of poles (counting multiplicities) is even. (It is $2(1-m)$ where $m$ is the genus of 8 .) Hence to show that the pole at the puncture has even order, it suffices to show that

(i) The number of poles of $d g / g$ on 8 is even.

(ii) The number of zeros of $d g / g$ on $\delta \backslash\{0\}$ is even.

(iii) The origin (if it is a zero of $d g / g$ ) is a zero of even order.

On $\delta, d g / g$ has poles precisely at the zeros and poles of $g$. Along $Z, g$ is unitary so no poles of $d g / g$ occur there. By the $180^{\circ}$ rotation $\rho_{Z}$ about $Z$, the zeros and poles of $g$ are paired, counting multiplicity. Hence, $d g / g$ has an even number of poles on 8 . This establishes (i). The zeros of $d g / g$ occur at branch points of $g$ (zeros of the Gauss curvature), and the multiplicity of the zero of $d g / g$ is equal to the branching order of $g$. Except for the origin, these zeros also occur in pairs (the point $p \in \delta \backslash Z$ being paired with $\rho_{Z}(p)$ and $q \in Z \backslash\{0\}$ being paired with $-q$.) This proves (ii). The tangent plane at the origin is the plane $P$ given by $x_{2}=0$. The $180^{\circ}$ rotations $\rho_{X}$ and $\rho_{Z}$ about $X$ and $Z$ are symmetries of $\delta$ and therefore also of $P \cap 8$. Thus $P \cap \delta$ must have an even number, say $2 k$, of curves passing through the origin. The order of branching of $g$ at the origin is then $2(k-1)$. This establishes (iii), completing the proof that $d g / g$ has a pole at infinity of even order.

The principal curvature function of a minimal surface is given by the expression ([HK97], page 15)

$$
k=\sqrt{-K}=\frac{4|d g / g| /|d h|}{(|g|+1 /|g|)^{2}} .
$$

Along $Z$, the tangent plane to $\delta$ is vertical, so $g$ is unitary and

$$
k=\frac{|d g / g|}{|d h|} .
$$

Since the curvature of $\delta$ is bounded by assumption and since (as we have already shown) $d h$ has a pole of order two at the end, we see from (18) that $d g / g$ has a pole at infinity of order at most two. Since the order is even, it must be exactly two. 


\section{Asymptotic behavior of symmetric, periodic, properly embedded minimal surfaces with finite topology and one end}

In this section we give estimates for the rate that a minimal graph with certain helicoidal qualities actually converges to a helicoid. We will use the estimates to prove that the periodic examples constructed in [HW08] are asymptotic to the helicoid. The estimates of Proposition 5.1 were also used in Section 3, to prove that periodic examples with small twist angles do not exist.

Proposition 5.1. Let $v(r, \theta)=\theta$, a function whose multigraph $S$ over $\mathbb{R}^{2} \backslash\{0\}$ is a half-helicoid of $H$. Suppose $S^{\prime}$ is another minimal multigraph of a function u over a region of the form

$$
W_{A}=\{(r, \theta): r \geq A,|\theta|<h\}
$$

with the property that for $r \geq A$,

$$
u(r, \pm h)=v(r, \pm h)= \pm h .
$$

Suppose further that $S^{\prime}$ has asymptotically vertical normals as $R \rightarrow \infty$. If $w=u-v$ is bounded, then

$$
\begin{aligned}
|w| & =o\left(r^{-\beta}\right), \\
|D w| & =o\left(r^{-(1+\beta)}\right), \\
\left|D^{2} w\right| & =o\left(r^{-(2+\beta)}\right)
\end{aligned}
$$

for any $\beta<\pi / 2 h$, where $D=\left(\frac{\partial}{\partial x_{1}}, \frac{\partial}{\partial x_{2}}\right)$, and $x_{1}=r \cos \theta, x_{2}=r \sin \theta$. In particular,

$$
\left|\frac{\partial w}{\partial \theta}\right|=o\left(r^{-\beta}\right), \quad \text { and } \quad\left|\frac{\partial^{2} w}{\partial \theta^{2}}\right|=o\left(r^{-\beta}\right)
$$

for any $\beta<\pi / 2 h$.

The proof of Proposition 5.1 involves a Phragmén-Lindelöf-type argument. To apply it, we first show that $w$ is the solution of a linear elliptic equation.

Lemma 5.2. The vertical distance function between two minimal graphs satisfies a linear elliptic equation

This is a special case of a well known result for quasilinear, elliptic partial differential equations. (See Gilbarg-Trudinger, [GT98], Chapter 10.) For the reader's convenience we include a proof of the lemma.

Proof of Lemma 5.2. A function $u$ whose graph is a minimal surface satisfies

$$
Q(u)=\left(1+u_{2}^{2}\right) u_{11}+\left(1+u_{1}^{2}\right) u_{22}-\left(2 u_{1} u_{2}\right) u_{12}=0 .
$$


If $v$ is another function whose graph is a minimal surface, then writing $w=u-v$,

$$
\begin{aligned}
0= & Q u-Q v \\
= & \left(1+u_{2}^{2}\right) w_{11}+\left(1+u_{1}^{2}\right) w_{22}-\left(2 u_{1} u_{2}\right) w_{12} \\
& \quad+v_{11}\left(u_{2}^{2}-v_{2}^{2}\right)+v_{22}\left(u_{1}^{2}-v_{1}^{2}\right)-2 v_{12}\left(u_{1} u_{2}-v_{1} v_{2}\right) \\
= & a_{i j} w_{i j}+\left[v_{11}\left(u_{2}+v_{2}\right)\right] w_{2}+\left[v_{22}\left(u_{1}+v_{1}\right)\right] w_{1} \\
& \quad-v_{12}\left(\left(u_{2}+v_{2}\right) w_{1}+\left(v_{1}+u_{1}\right)\right) w_{2} \\
= & a_{i j} w_{i j}+\left[v_{22}\left(u_{1}+v_{1}\right)-v_{12}\left(u_{2}+v_{2}\right)\right] w_{1} \\
& \quad+\quad\left[v_{11}\left(u_{2}+v_{2}\right)-v_{12}\left(u_{1}+v_{1}\right)\right] w_{2} \\
= & a_{i j} w_{i j}+b_{k} w_{k},
\end{aligned}
$$

where

$$
\begin{aligned}
& a_{11}=1+u_{2}^{2}, a_{22}=1+u_{1}^{2}, a_{12}=a_{21}=u_{1} u_{2}, \\
& b_{1}=v_{22}\left(u_{1}+v_{1}\right)-v_{12}\left(u_{2}+v_{2}\right), \\
& b_{2}=v_{11}\left(u_{2}+v_{2}\right)-v_{12}\left(u_{1}+v_{1}\right) .
\end{aligned}
$$

The operator $L$, defined by

$$
L w:=a_{i j} w_{i j}+b_{k} w_{k}=0,
$$

is elliptic and linear (its coefficients do not depend on $w$ or its derivatives), and $L w=0$.

Suppose $u_{n}$ and $v_{n}$ are sequences of solutions to the minimal surface equation on a domain $\Omega$. Then $w_{n}=u_{n}-v_{n}$ satisfies $L_{n} w_{n}=0$, where $L_{n}=L\left(u_{n}, v_{n}\right)$ is the linear elliptic operator defined in Lemma 5.2. We will have need of the following result in the proof of Proposition 5.1.

Corollary 5.3. If $u_{n}$ and $v_{n}$ converge uniformly to zero, then $L_{n}$ converges smoothly to the Laplacian on compact subsets of $\Omega$.

Proof. Let $f_{i}: \Omega \rightarrow \mathbb{R}$ be a sequence of solutions to the minimal surface equation that converge uniformly to zero, and let $K$ be a compact subset of $\Omega$. Fix a positive integer $k$ and let

$$
\Lambda=\limsup _{i}\left(\max _{x \in K}\left|D^{k} f_{i}(x)\right|\right) .
$$

By passing to a subsequence if necessary we may assume that the lim sup is a limit. By [GT98], Corollary 16.7, $\left\|f_{i}\right\|_{C^{k+1}}$ is uniformly bounded on compact subsets of $\Omega$. Thus, by passing to a further subsequence if necessary, we may assume that the $f_{i}$ converge, on compact subsets in the $C^{k}$ norm, to a limit function $f$. But since the $f_{i}$ converge uniformly to zero, $f$ is the zero function. Hence $\Lambda=0$. 
We now apply the conclusion of the previous paragraph to the sequences $u_{n}$ and $v_{n}$. From (21), it follows that $L_{n}$ converges smoothly on compact subsets to the Laplacian.

Proof of Proposition 5.1. Let $w=u-v$ be the difference between the two functions that define the minimal multigraphs on $W_{A}$. The function $w$ is zero on the rays in $\partial W_{A}$ and bounded above on the circular arc of radius $A$ in $\partial W_{A}$. By Lemma 5.2, $w$ satisfies $L w=0$ for the linear elliptic operator $L$ defined in (22).

For any $0<\beta<\alpha<\frac{\pi}{2 h}$, the functions

$$
\begin{aligned}
& f(r, \theta)=r^{-\beta} \cos (\alpha \theta), \\
& g(r, \theta)=r^{\beta} \cos (\alpha \theta)
\end{aligned}
$$

are both positive on $\overline{W_{A}}$, and they satisfy

$$
\begin{gathered}
\Delta f=\left(\beta^{2}-\alpha^{2}\right) r^{-2} f<0, \\
\Delta g=\left(\beta^{2}-\alpha^{2}\right) r^{-2} g<0
\end{gathered}
$$

on $W_{A}$.

Claim. There exists an $A^{\prime} \geq A$, such that

$$
L f<0 \quad \text { and } \quad L g<0 \quad \text { on } W_{A^{\prime}}=\left\{(r, \theta): r \geq A^{\prime},|\theta|<h\right\} .
$$

Proof of Claim. On $S$, the half helicoid that is the multigraph of $v(r, \theta)=\theta$, the normal is asymptotically vertical as $r \rightarrow \infty$, and by assumption the same is true for $S^{\prime}$, the graph of $u(r, \theta)$. Therefore, $|D u| \rightarrow 0$ and $|D v| \rightarrow 0$ as $r \rightarrow \infty$. Moreover it is elementary to calculate that $|D v|=O\left(r^{-1}\right)$ and $\left|D^{2} v\right|=O\left(r^{-2}\right)$.

From (21), (23), and the preceding paragraph, we may compute

$$
\begin{aligned}
L f & =\Delta f+u_{2}^{2} f_{11}+u_{1}^{2} f_{22}-2 u_{1} u_{2} f_{12}+b_{1} f_{1}+b_{2} f_{2} \\
& =C r^{-2} f+o(1)\left(f_{11}+f_{22}-2 f_{12}\right)+O\left(r^{-2}\right)\left(f_{1}+f_{2}\right),
\end{aligned}
$$

where $C=\left(\beta^{2}-\alpha^{2}\right)<0$, and the $b_{k}$ are defined in (21). It is straightforward to compute that $|D f|=O\left(r^{-1}\right) f$ and $\left|D^{2} f\right|=O\left(r^{-2}\right) f$, from which it follows that

$$
\begin{aligned}
L f & =C r^{-2} f+o(1) O\left(r^{-2}\right) f+O\left(r^{-3}\right) f \\
& =f\left[C r^{-2}+o(1) O\left(r^{-2}\right)+O\left(r^{-3}\right)\right] .
\end{aligned}
$$

Since $f>0$ on $W_{A}$ and $C<0$, it follows from (25) that $L f<0$ for $r$ sufficiently large.

An almost identical proof establishes that $L g<0$ for $r$ sufficiently large. 
Let $A^{\prime}$ be the constant whose existence is established by Claim 1. Since $w$ is bounded on $\overline{W_{A^{\prime}}}$ and $f$ is strictly positive on $\overline{W_{A^{\prime}}}$, there exists a $\lambda>0$ large enough so that $\lambda f>w$ on the circular arc in $\partial W_{A^{\prime}}$ of radius $A^{\prime}$. On the rays in $\partial W_{A^{\prime}}, w$ is identically zero and both $f$ and $g$ are strictly positive. Therefore, for any $\epsilon>0$,

$$
\tilde{w}_{\epsilon}:=w-\lambda f-\epsilon g<0 \quad \text { on } \partial W_{A^{\prime}} .
$$

Also, for any $\epsilon>0, \tilde{w}_{\epsilon}(r, \theta)$ is negative for $r$ sufficiently large. (This is because $w$ is bounded, $f \rightarrow 0$ and $g \rightarrow \infty$ as $r \rightarrow \infty$.) From Claim 1 we have

$$
L \tilde{w}_{\epsilon}>0 \text { on } W_{A^{\prime}} .
$$

By the maximum principle, we may conclude that $\tilde{w}_{\epsilon}<0$ on $W_{A^{\prime}}$. Since this is valid for any positive $\epsilon$, it follows that $w \leq \lambda f$ on $W_{A^{\prime}}$, which implies that for any $\beta<\frac{\pi}{2 h}$,

$$
w \leq \lambda r^{-\beta} \text {. }
$$

We can repeat the same argument for $-w$ and conclude that $-w \leq \lambda r^{-\beta}$. We have proved the first equation of (19) for any $\beta<\frac{\pi}{2 h}$. In particular, $S$ is asymptotic to $S^{\prime}$.

It remains to establish the asymptotic decay rates, stated in (19), of $D w$ and $D^{2} w$. If a surface is minimal, then its image under rescaling of $\mathbb{R}^{3}$ is also minimal. Therefore

$$
u_{R}(p):=\frac{u(R p)}{R} \quad \text { and } \quad v_{R}(p):=\frac{v(R p)}{R}
$$

are solutions of minimal surface equation defined for $r>A / R$ and $|\theta| \leq h$. By Schwartz reflection in the boundary rays, we may extend $u_{R}$ and $v_{R}$ to the region defined by $r>A / R$ and $|\theta| \leq 2 h$. Moreover, $u_{R} \rightarrow 0$ and $v_{R} \rightarrow 0$ as $R \rightarrow \infty$. By Lemma 5.2 and Corollary 5.3, $w_{R}=u_{R}-v_{R}$ satisfies a linear elliptic equation, $L_{R} w_{R}=0$, and as $R \rightarrow \infty, L_{R}$ converges smoothly to the Laplacian on compact subsets of the region where $r>0$ and $|\theta|<2 h$.

Let $\Gamma$ be the arc given by $r=1$ and $|\theta| \leq h$. Let $\Omega$ be the open set defined by $1 / 2<r<2$ and $|\theta| \leq \frac{3}{2} h$. We will apply the Schauder interior estimates ([GT98], Theorem 6.2) for the operator $L_{R}$ on $\Omega$. Since $L_{R}$ is converging smoothly on $\Omega$, there exist positive constants $C$ and $R^{*}$ such that for $R>R^{*}$ :

$$
\begin{gathered}
\sup _{p \in \Gamma}\left|D w_{R}(p)\right| \leq C \sup _{p \in \Omega}\left|w_{R}(p)\right|, \\
\sup _{p \in \Gamma}\left|D^{2} w_{R}(p)\right| \leq C \sup _{p \in \Omega}\left|w_{R}(p)\right| .
\end{gathered}
$$

Since $w_{R}(p)=\frac{w(R p)}{R}$, the first and second derivatives of $w_{R}$ satisfy

$$
\begin{aligned}
D w_{R}(p) & =D w(R p), \\
D^{2} w_{R}(p) & =R D^{2} w(R p) .
\end{aligned}
$$


Let $R \Gamma:=\{R p: p \in \Gamma\}$ and $R \Omega:=\{R p: p \in \Omega\}$. It now follows from (27) and (28) that for $R>R^{*}$ :

$$
\begin{array}{r}
\sup _{q \in R \Omega}|D w(q)| \leq R^{-1} C \sup _{q \in R \Omega}|w(q)|, \\
R \sup _{q \in R \Omega}\left|D^{2} w(q)\right| \leq R^{-1} C \sup _{q \in R \Omega}|w(q)| .
\end{array}
$$

Since we have already established that $w=o\left(r^{-\beta}\right)$, the last two estimates of (19) follow immediately from (29). The bounds (20) follow directly from (19).

We will use Proposition 5.1 to prove the main result of this section, which concerns the asymptotic behavior of ends of embedded screw-motion-invariant minimal surfaces with some of the properties of the surfaces constructed in [HW08].

Proposition 5.4. Let $S \subset \mathbb{R}^{3}$ be a properly embedded minimal surface that is invariant under a screw motion

$$
\sigma:(r \cos \theta, r \sin \theta, \theta) \mapsto(r \cos (\theta+\beta), r \sin (\theta+\beta), \theta+t)
$$

with $t \neq 0$. Suppose that the slope of the tangent plane tends to 0 as $r=\sqrt{x^{2}+y^{2}}$ tends to infinity, that the intersection of $S$ with some horizontal plane coincides with a line L outside of a compact set, and that the two ends of the line L correspond to different ends of $S / \sigma$.

Then $S$ is asymptotic to a helicoid $H^{\prime}$ with axis $Z$. Indeed, the vertical distance between $S$ and $H^{\prime}$ decays faster than $r^{-\beta}$ for every

$$
\beta<\left|\frac{p}{t}\right| \pi
$$

where $p$ is the pitch of the helicoid $H^{\prime}$.

The pitch of $H^{\prime}$ may be defined as the (constant) value that $\partial z / \partial \theta$ takes on $H^{\prime} \backslash Z$. Note that if $\beta=t=2 h$, then $\sigma$ is the screw motion $\sigma_{2 h}$ used elsewhere in this paper (3).

Remark 5.5. The theorem is also true for surfaces $S$ with boundary, provided the boundary lies within a bounded distance of $Z$.

Proof. By translation and rotation, we may assume that the line $L$ is $X$. We will allow $S$ to have boundary as indicated in the remark. Let $C$ be the interior of a closed solid cylinder about $Z$ that contains $\partial S$. Choose the radius $R$ of the cylinder large enough that the tangent planes to $S \backslash C$ are all nearly horizontal, and so that $S \cap\{z=0\}$ coincides with $X$ outside of $C$. We may assume that $S \subset \mathbb{R}^{3} \backslash C$ and that $\partial S \subset \partial C$; otherwise replace $S$ by $S \backslash C$. 
Let $X^{+}$and $X^{-}$denote the positive and negative portions of $X \backslash C$ :

$$
\begin{aligned}
& X^{+}=\{(x, 0,0): x \geq R\}, \\
& X^{-}=\{(x, 0,0): x \leq-R\} .
\end{aligned}
$$

Thus we have

$$
S \cap\{z=0\}=X^{+} \cup X^{-} .
$$

Note that each component $V$ of $S$ is a covering space of $\mathbb{R}^{2} \backslash \mathbb{B}(0, R)$. Since $V$ is embedded, it must be either a single-sheeted covering or an infinite covering. Thus $V$ can be parametrized as

$$
(r, \theta) \mapsto(r \cos \theta, r \sin \theta, f(r, \theta)) \quad(r \geq R, \theta \in \mathbb{R})
$$

where this map is either periodic (with period $2 \pi$ ) or else one-to-one, according to whether $V$ is a single-sheeted or not. Note that if $f$ is not periodic, then by properness $f$ is not bounded above or below.

Now let $V$ be the component of $S$ containing $X^{+}$. By the hypothesis about the ends of $L, V$ cannot be the component of $S$ that contains $X^{-}$, nor can it be the component that contains any of the rays identified with $X^{-}$in $S / \sigma$. Thus

$$
V \cap \sigma^{n} X^{-}=\emptyset \quad \text { for } n \in \mathbb{Z} .
$$

Note that we can parametrize $V$ as in (32) with a function $f$ satisfying

$$
f(r, 0) \equiv 0 .
$$

By Schwartz reflection, $f(r,-\theta) \equiv-f(r, \theta)$. In particular, $f(r,-\pi)=-f(r, \pi)$. Now $f(r, \pi) \neq 0$ since $V$ is disjoint from $X^{-}$. Thus $f(r, \pi) \neq f(r,-\pi)$, so $f(r, \theta)$ does not have period $2 \pi$ and therefore is not periodic.

It follows (from (31)) that $f(r, \theta)=0$ if and only if $\theta=0$.

By reflecting in the plane $\{z=0\}$, if necessary, we may assume that $f(r, \theta)>0$ for $\theta>0$. We may also assume that $t>0$. (Otherwise replace $\sigma$ by $\sigma^{-1}$.) Since $f$ is not bounded above, it must intersect the plane $\{z=t\}$. By (31) and by the $\sigma$-invariance,

$$
S \cap\{z=t\}=\sigma X^{+} \cup \sigma X^{-} .
$$

Thus $V \cap\{z=t\}$ must be one or both of the rays $\sigma X^{ \pm}$. By (33), it must be the ray $\sigma X^{+}$. Let $\Theta$ be the value of the parameter $\theta$ corresponding to this ray. Then we have:

$$
\begin{aligned}
& 0<f(r, \theta)<t \quad \text { for } 0<\theta<\Theta, \\
& f(r, 0) \equiv 0, \quad \text { and } \quad f(r, \Theta) \equiv t .
\end{aligned}
$$


If we dilate the surface $S$ by $\lambda>0$, then $R, t$, and $f(r, \theta)$ get replaced by $\lambda R, \lambda t$, and $f(r / \lambda, \theta)$, but $\Theta$ does not change.

Thus by scaling by $\lambda=\Theta / t$, we can assume that $t=\Theta$. Hence $\sigma=\sigma_{2 h}$ (see (3)), where $h=t / 2$. Now $V$ (or more precisely $\sigma_{-h} V$ ) satisfies the hypotheses of Proposition 5.1. Hence $V$ is asymptotic to $H$ with the asserted decay rate.

Let $W$ be the component of $S$ containing $X^{-}$. Exactly the same argument shows that $W$ is also asymptotic (with the asserted decay rate) to some helicoid $H^{\prime}$ with axis $Z$ and containing $X$. Note that $H^{\prime}=H$ since otherwise $V$ and $W$ would intersect.

Finally, $S$ can have no component other than $V$ and $W$, because any such component, being trapped between $V$ and $W$, would have to intersect the plane $\{z=0\}$, and by (31) the only possible intersections are $X^{+}$and $X^{-}$.

\section{References}

[CM04] T. H. Colding and W. P. Minicozzi, II, The space of embedded minimal surfaces of fixed genus in a 3-manifold. III. Planar domains. Ann. of Math. (2) 160 (2) (2004), 523-572. Zbl 1076.53068 MR 2123932

[EWW02] T. Ekholm, B. White, and D. Wienholtz, Embeddedness of minimal surfaces with

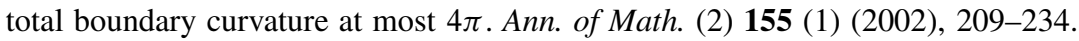
Zbl 1017.53013 MR 1888799

[GT98] D. Gilbarg and N. S. Trudinger, Elliptic Partial Differential Equations of Second Order. 3rd edition, Grundlehren Math. Wiss. 224 Springer-Verlag, Berlin 1998. Zbl 0562.35001 MR 0737190 MR 1814364

[HR90] R. Hardt and H. Rosenberg, Open book structures and unicity of minimal submanifolds. Ann. Inst. Fourier (Grenoble) 40 (3) (1990), 701-708. Zbl 0702.53039 MR 1091838

[HK97] D. Hoffman and H. Karcher, Complete embedded minimal surfaces of finite total curvature. In Encyclopaedia Math. Sci. 90, R. Osserman, ed., Springer-Verlag, Berlin 1997, 5-93. Zbl 0890.53001 MR 1490038

[HKW99] D. Hoffman, H. Karcher, and F. Wei, The singly periodic genus-one helicoid. Comment. Math. Helv. 74 (1999), 248-279. Zbl 0958.53006 MR 1691949

[HM03] D. Hoffman and J. McCuan, Embedded minimal ends asymptotic to the helicoid. Comm. Anal. Geom. 11 (2003), 721-735. Zbl 1079.53016 MR 2015173

[HW08] D. Hoffman and B. White, Genus-one helicoids from a variational point of view. Comment. Math. Helv. 83 (2008), 767-813. Zbl 05361852 MR 2442963

[LM83] L. P. Jorge and W. H. Meeks, III, The topology of complete minimal surfaces of finite total Gaussian curvature. Topology 22 (1983), 203-221. Zbl 0517.53008 MR 683761

[MR93] W. H. Meeks, III and H. Rosenberg, The geometry of periodic minimal surfaces. Comment. Math. Helv. 68 (4) (1993), 538-578. Zbl 0807.53049 MR 1241472

[MR05] W. H. Meeks, III and H. Rosenberg, The uniqueness of the helicoid. Ann. of Math. 161 (2005), 727-758. Zbl 1102.53005 MR 2153399 
[MY82] W. H. Meeks, III and S. T. Yau, The existence of embedded minimal surfaces and the problem of uniqueness. Math. Z. 179 (1982), 151-168. Zbl 0479.49026 MR 0645492

[Nit73] J. C. C. Nitsche, A new uniqueness theorem for minimal surfaces. Arch. Rational Mech. Anal. 52 (1973), 319-329. Zbl 0285.49001 MR 0341258

[Nit89] J. C. C. Nitsche, Lectures on Minimal Surfaces. Volume 1, Cambridge University Press, Cambridge 1989. Zbl 0688.53001 MR 1015936

[Oss63] R. Osserman, On complete minimal surfaces. Arch. Rational Mech. Anal. 13 (1963), 392-404. Zbl 0127.38003 MR 0151907

[Oss86] R. Osserman, A Survey of Minimal Surfaces. 2nd edition, Dover Publications, New York 1986. Zbl 0209.52901 MR 0852409

[RR98] L. Rodríguez and H. Rosenberg, Minimal surfaces in $\mathbf{R}^{3}$ with one end and bounded curvature. Manuscripta Math. 96 (1) (1998), 3-7. Zbl 0968.53009 MR 1624411

[Sch83] R. M. Schoen, Uniqueness, symmetry, and embeddedness of minimal surfaces. $J$. Differential Geom. 18 (1983), 791-809. Zbl 0575.53037 MR 730928

[WHW06] M. Weber, D. Hoffman, and M. Wolf, An embedded genus-one helicoid. Ann. of Math. 169 (2009), 1-102. MR 2480608

Received July 16, 2007

David Hoffman, Department of Mathematics, Stanford University, Stanford, CA 94305, U.S.A.

E-mail: hoffman@math.stanford.edu

Brian White, Department of Mathematics, Stanford University, Stanford, CA 94305, U.S.A.

E-mail: white@math.stanford.edu 